\title{
Research on transmittance / reflectance test system of optical lens based on differential measurement \\ Lixia Shi ${ }^{1, a}$, Lixin Yang ${ }^{1, b^{*}}$ and Ming Liu ${ }^{1, c}$ \\ ${ }^{1}$ The School of Changchun University of Science and Technology,No.7186 Weixing Road, Changchun130022, China

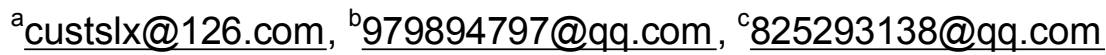

Keywords: transmittance; reflectance; double optical paths; double-integrating spheres.

Abstract. We designed an optical lens transmittance/ reflectance test system, which can measure both the transmittance and the reflectance of the sample under different incident angles at the same time, based on differential measurement. This paper elaborates the system composition and working principle, and demonstrates it by experiments. The experiments show that the measurement accuracy of the transmittance/ reflectance of white light can reach $0.5 \%$, and that of infrared band can reach $1 \%$.

\section{Introduction}

The transmittance and reflectance are very important parameters for evaluating the performance of optical components. As a result, there have been a lot of devices that can be used to measure transmittance or reflectance. However, the devices which can simultaneously measure the transmittance and reflectance are very rare. And the measurement accuracy of the equipment is very low.

For traditional transmittance and reflectance measurements, they usually use a single optical path, which lead to high demand of the environment and low measurement accuracy. This system uses dual-optical paths to measure, and the double-integrating spheres structure makes the simultaneously measurement of the transmittance and reflectance possible. It has a very high degree of automation.

\section{Measuring principle of the system}

Transmittance of a surface, denoted $\mathrm{T}$, is defined as:

$$
\mathrm{T}=\frac{\mathrm{I}_{1}}{\mathrm{I}_{0}} \times 100 \% .
$$

Where,

$I_{1}$ is the intensity of the light transmitted by that surface;

$\mathrm{I}_{0}$ is the intensity of the light received by that surface.

Reflectance of a surface, denoted $\mathrm{R}$, is defined as:

$$
\mathrm{R}=\frac{\mathrm{I}_{2}}{\mathrm{I}_{0}} \times 100 \% .
$$

Where,

$\mathrm{I}_{2}$ is the intensity of the light reflected by that surface;

$\mathrm{I}_{0}$ is the intensity of the light received by that surface.

In the system, the light intensity is converted into electrical current through the detector. And then the post-processing circuit translates the current signal to voltage signal. $U_{1}$ is the voltage of the optical path for measurement, $\mathrm{U}_{2}$ is the voltage of the optical path for reference. 


$$
\mathrm{T}=\frac{\mathrm{U}_{1}}{\mathrm{U}_{2}} \times 100 \%
$$

We have to carry out the measurement multiple times. Because the asymmetric of the two optical paths will affect the measurement results.

$$
\mathrm{K}=\frac{\mathrm{U}_{2}^{\prime}}{\mathrm{U}_{1}^{\prime}} \times 100 \% .
$$

Where,

$U_{1}^{\prime}$ is the voltage of the optical path for measurement when the sample surface is not put in it; $U_{2}^{\prime}$ is the voltage of the optical path for reference when the sample surface is not put in it.

In this system, the measurement formula of transmittance is:

$$
\mathrm{T}=\mathrm{K} \times \frac{\mathrm{U}_{1}}{\mathrm{U}_{2}} \times 100 \% .
$$

The principle of the measurement formula of reflectance is the same as that of transmittance, so it is not listed here.

\section{System composition}

The structure and composition of this system is shown in Figure 1. It consists of a light source, an intermediate optical path, a receiving unit and a signal processing unit.

Light source. The spectral range of this system is between $300-1600 \mathrm{~nm}$. The bromine tungsten light's spectral range $(300 \sim 2500 \mathrm{~nm})$ meets the requirements, and has high luminous efficiency, so we use bromine tungsten lamp as light source.

One shaft of light emitted by a tungsten filament lamp is used as the white light source after being collimated.

Another shaft of light emitted by a tungsten filament lamp form a particular wavelength of monochromatic light required by the system through a monochromator. Then it goes through a collimation lens and transforms into parallel light[1].

For the grating, the chromatic resolving power and beam splitting ability is proportional to the spectral class, but the light intensity distribution is inversely proportional to the spectral class, which makes the zero-order fringe who takes a large proportion of the light intensity in the use of grating useless. To improve the energy efficiency of the system, we choose the blazed grating. According to the requirements, we use 2 blazed gratings whose blaze wavelength are $500 \mathrm{~mm}$ and $1250 \mathrm{~mm}$ respectively to split the light from visible to near infrared spectral range.

The 2 light sources are switched by moving the mirror.

Intermediate optical path. The Beam diameter required is $10 \mathrm{~mm}$, but the collimated beam diameter is larger than that, so we place a diaphragm there.

We use a half transparent and half reflecting mirror because we need to split the light collimated. The light transmitted is used for measurement. The light reflected by the sample will be reflected by a reflector and then be used for reference.

This system uses a lock-in amplifier to reduce the influence of stray light, so the light beam need to be modulated. In order to detect the voltage of the light respectively in the phase lock loop section, we need to modulate the two light beams in different frequency. So the chopper disk is designed to have an inner hole and an outer hole. The light for measurement goes into the integrating sphere directly after modulation, while the light for reference goes into the integrating sphere at the top after being 
coupled into the optical fiber by a condenser. The sample is placed on a bracket whose underpart is connected with the turntable. So the sample can receive the light beam from $0^{\circ}$ to $90^{\circ}[2]$.

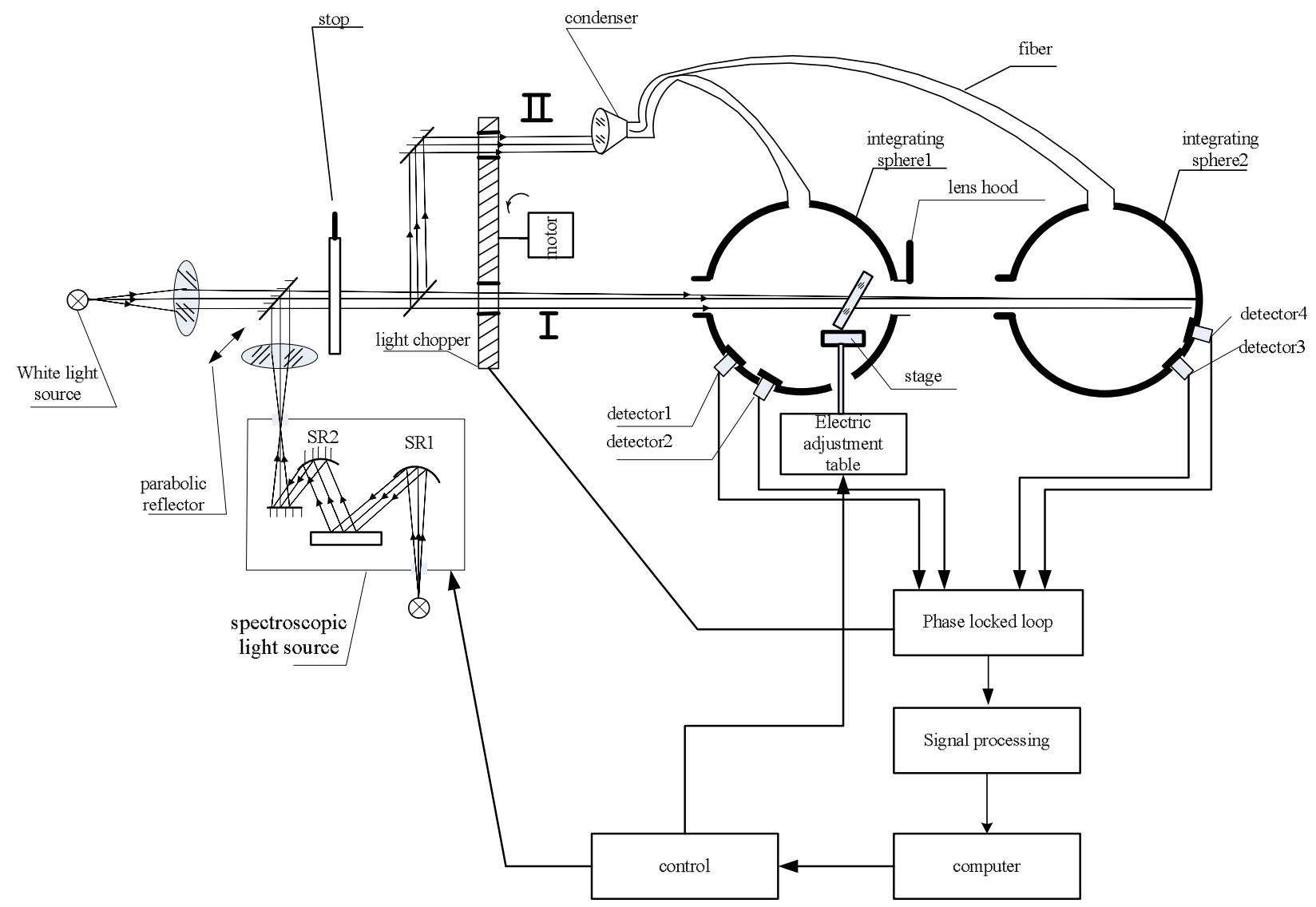

Fig.1 System Composition

Receiving unit.Integrating sphere can reduce the errors caused by the shape and divergence angle of the light, and the response differences in different locations on the detector, making measurements more reliable. So we choose the double-integrating spheres structure, shown in Figure 2. The first integrating sphere is used for measuring the reflectance, and the other is used for the transmittance[3].

Due to the wide spectral range, a dual detector is chosen. The light not more than $900 \mathrm{~nm}$ is received by silicon photovoltaic cells, and the other is received by the InGaAs detector.

signal processing unit. The signal processing is achieved by a lock-in amplifier, which is mainly composed of three parts: a signal channel, a reference channel and a correlator. The signal channel is used to enhance the measured signal, and filter out some interference and noise suppression. The reference channel is used signal shaping, frequency division and phase shift of the signal for reference. The correlator is composed of a phase-sensitive detector and an integrator. The phase sensitive detector is used to implement the function of the multiplier, and also to detect the phase of the two signals. The integrator is used to implement the integral function of relevant operation, and has the function of the low-pass filter. The 2 lock-in amplifier respectively transmit the in-phase amplitude and quadrature amplitude of the signal for measurement detected by the phase sensitive detector to the upper computer through the data acquisition card. At last we write the user interface using LabVIEW, as shown in Figure 3[4,5]. 

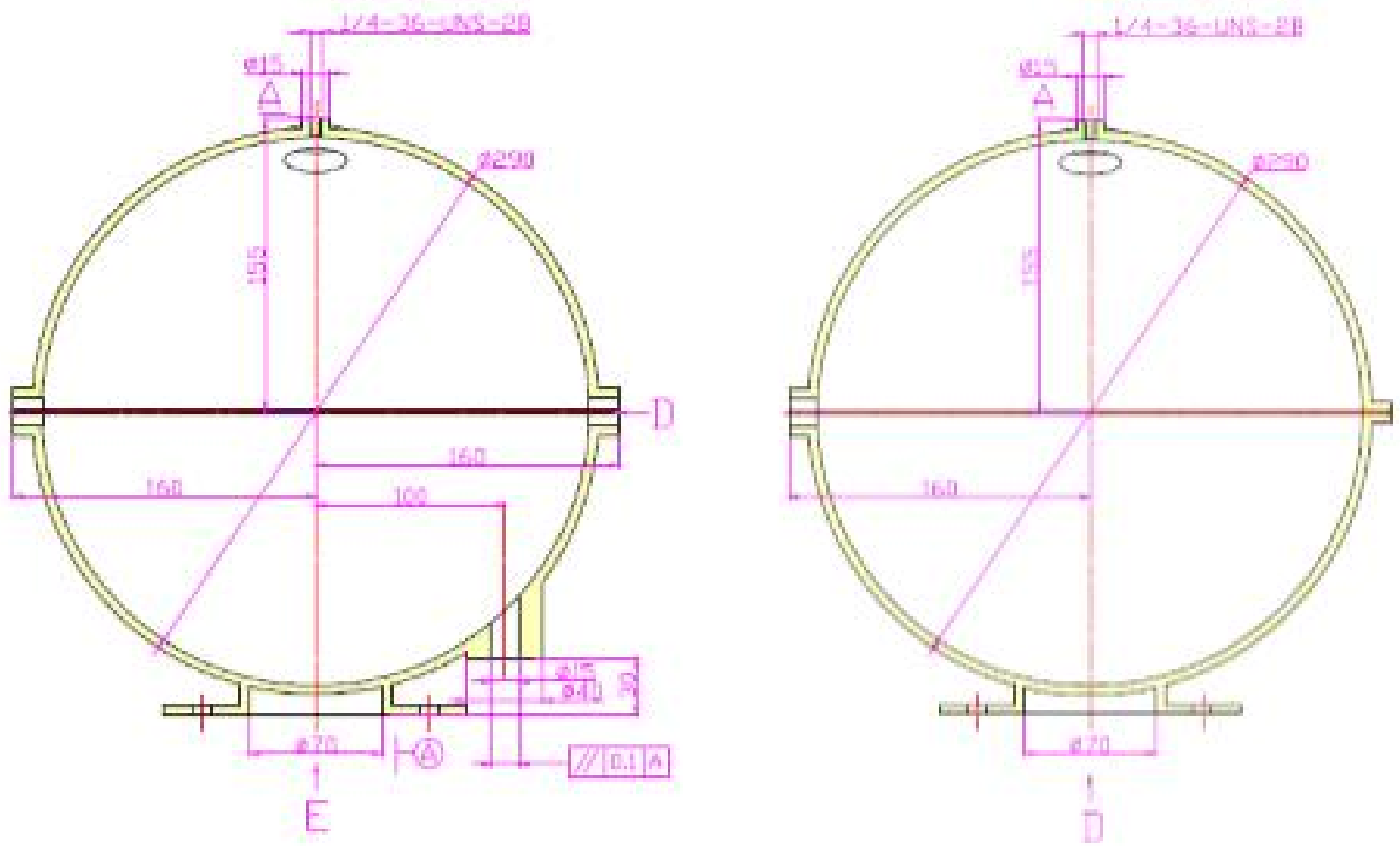

Fig.2 Receiving Unit

\section{Transmittance / Reflectance test system of optical lens}

\section{Transmittance}

0

Monochromator control

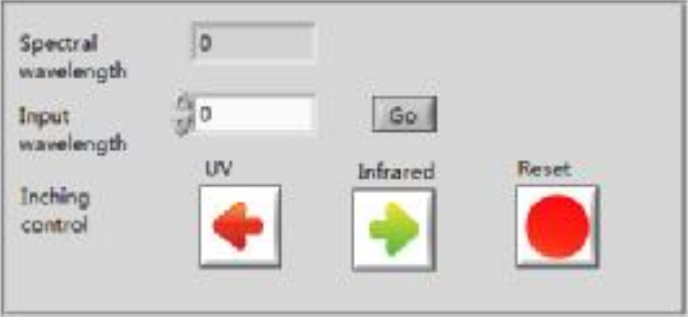

Communication settings

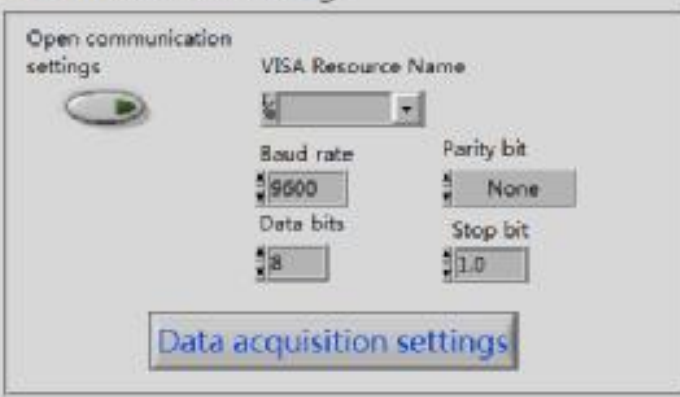

Reflectance

o

Mirror control

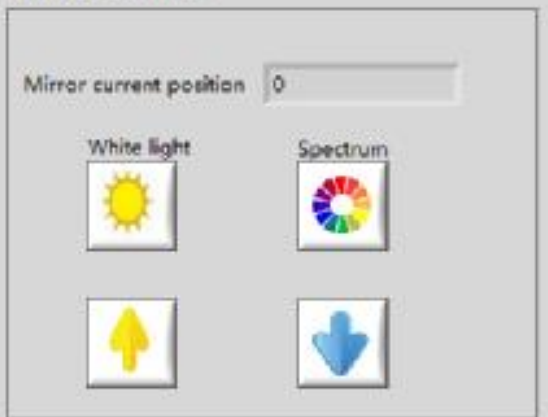

Sample control

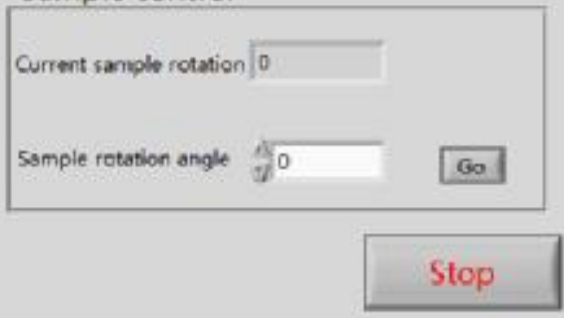

Fig.3 User Interface

The light goes through the spectroscope, the shaft transmitted is used for measurement, and the shaft reflected is used for reference after being reflected again by the reflector. The 2 shafts of light are modulated into different frequency respectively through the inner hole and the outer hole of the chopper disk. The light for measurement goes into the integrating sphere directly after modulation, while the light for reference goes into the integrating sphere at the top after being coupled into the optical fiber by a condenser. 
The first integrating sphere is used for measuring the reflectance. For reflectance measurement, we put the hood cover on its surface, then the light reflected will go to the detector after the uniform light effect by the integrating sphere. At the same time, the light for reference will go to the same detector after the uniform light effect by the integrating sphere. These 2 signals will then be transmitted to the computer after being processed by the lock-in amplifier. Finally, we can get the reflectance of the sample according to the calculation formula.

The second integrating sphere is used for measuring the transmittance. For transmittance measurement, we open the hood cover on its surface, then the light transmitted will go to the detector after the uniform light effect by the integrating sphere. At the same time, the light for reference will go to the same detector after the uniform light effect by the integrating sphere. In the same manner, we can get the transmittance of the sample.

\section{Experimental verification}

The experimental data of white light is shown in Table 1:

\begin{tabular}{lllllll}
\hline $\begin{array}{l}\text { Standard } \\
\text { value(\%) }\end{array}$ & Test1(\%) & Test2(\%) & Test3(\%) & Test4(\%) & Test5(\%) & $\begin{array}{l}\text { Limit } \\
\text { error } \\
(\%)\end{array}$ \\
\hline 90.36 & 90.05 & 90.10 & 90.13 & 90.08 & 90.12 & 0.31 \\
60.52 & 60.26 & 60.33 & 60.35 & 60.20 & 60.37 & 0.32 \\
30.44 & 30.16 & 30.08 & 30.07 & 30.30 & 30.24 & 0.37 \\
10.25 & 10.11 & 10.18 & 10.04 & 10.10 & 10.06 & 0.21 \\
\hline
\end{tabular}

Table 1 Experimental Data of White Light

The experimental data of the transmittance of $1064 \mathrm{~nm}$ is shown in Table 2:

\begin{tabular}{lllllll}
\hline $\begin{array}{l}\text { Standard } \\
\text { value(\%) }\end{array}$ & Test1(\%) & Test2(\%) & Test3(\%) & Test4(\%) & Test5(\%) & $\begin{array}{l}\text { Limit } \\
\text { error } \\
(\%)\end{array}$ \\
\hline 90.36 & 90.01 & 89.93 & 89.98 & 90.04 & 89.90 & 0.46 \\
60.52 & 60.01 & 60.05 & 60.10 & 59.97 & 60.08 & 0.55 \\
30.44 & 30.03 & 29.98 & 29.93 & 30.01 & 29.95 & 0.51 \\
10.25 & 9.81 & 9.89 & 9.93 & 9.90 & 9.92 & 0.44 \\
\hline
\end{tabular}

Table 2 Experimental Data of the Transmittance of $1064 \mathrm{~nm}$

The biggest error of the transmittance of white light is less than $0.4 \%$; that of infrared band is less than $0.6 \%$.

\section{Conclusions}

The experimental results show that the biggest error of the transmittance of white light is less than $0.5 \%$; that of infrared band is less than $1 \%$. The system can reach the design requirements. Since I didn't get the reflectance standard of the sample, I mainly measured the transmittance.

This testing device is a brand new system. It can get rid of the high demand of the background light which other systems need. And it uses the lock-in amplifier technology, which reduces the noise of background signals in the traditional test, and improves the measurement accuracy. The system also uses a light transmitting optical fiber, filter spectral calibration, integrating sphere photocell receiving and so on, as far as possible to improve the accuracy of the measurement.

\section{References}

[1] WANG Jin-song,HUI Fei,AN Zhi-yong Research on the Spectrumtransmissivity Detection System of the Optical Sight[J]. Science Technology and Engineering,2010,33:8259-8261+8276. (in Chinese) 
[2] XIE Yun, LIU Jun, LI Hai-feng, LIU Xu, GU Pei-fu. Measuring spectral reflectance and transmittance of heavy caliber optical thin-film[J]. Optical Instruments,2004,02:164-167.(in Chinese)

[3] LI Chen-xi,ZHAO Hui-juan,WANG Qiu-yin,XU Ke-xin,SONG Yang,ZHANG Wen-jun. A Near Infrared Multi-wavelength Optical Properties Measurement SystemBased on

Double-integrating-spheres and Lock-in Detection[J]. Acta Photonica Sinica,2009,07:1811-1815.(in Chinese)

[4] CHEN Dong,HUANG Yun,ZHANG Bin,YANG Kai-yong. Design of transmissivity and reflectivity automatic measuring system [J]. Optical Technique,2007,S1:210-211+213.(in Chinese) [5] LIU Wen-zhi HUANG Yun YANG Kai-yong. The automatie measuring system of differential transmissivit yandreflectiviyt measurer based on LabView [J]. Infrared and Laser Engineering,2006,S2:119-122.(in Chinese) 